ARTICLE

DOI: $10.1038 / \mathrm{s} 41467-018-05950-7$

\title{
A molecular overlayer with the Fibonacci square grid structure
}

\author{
Sam Coates (i) ${ }^{1}$, Joseph A. Smerdon ${ }^{2}$, Ronan McGrath ${ }^{1} \&$ Hem Raj Sharma (iD ${ }^{1}$
}

Quasicrystals differ from conventional crystals and amorphous materials in that they possess long-range order without periodicity. They exhibit orders of rotational symmetry which are forbidden in periodic crystals, such as five-, ten-, and twelve-fold, and their structures can be described with complex aperiodic tilings such as Penrose tilings and Stampfli-Gaehler tilings. Previous theoretical work explored the structure and properties of a hypothetical four-fold symmetric quasicrystal-the so-called Fibonacci square grid. Here, we show an experimental realisation of the Fibonacci square grid structure in a molecular overlayer. Scanning tunnelling microscopy reveals that fullerenes $\left(C_{60}\right)$ deposited on the two-fold surface of an icosahedral Al-Pd-Mn quasicrystal selectively adsorb atop $\mathrm{Mn}$ atoms, forming a Fibonacci square grid. The site-specific adsorption behaviour offers the potential to generate relatively simple quasicrystalline overlayer structures with tunable physical properties and demonstrates the use of molecules as a surface chemical probe to identify atomic species on similar metallic alloy surfaces.

\footnotetext{
${ }^{1}$ Surface Science Research Centre and Department of Physics, University of Liverpool, Liverpool L69 3BX, UK. ${ }^{2}$ Jeremiah Horrocks Institute for Mathematics, Physics and Astronomy, University of Central Lancashire, Preston PR1 2HE, UK. Correspondence and requests for materials should be addressed to H.R.S. (email: h.r.sharma@liv.ac.uk)
} 
uasicrystalline phases have been observed in a range of materials, including intermetallics ${ }^{1}$, liquid crystals $^{2}$, polymers $^{3,4}$, colloids ${ }^{5,6}$, perovskites ${ }^{7}$ and overlayer structures of single elements ${ }^{8-11}$ and molecules ${ }^{12,13}$. All of these quasicrystalline bulk and epitaxial phases exhibit forbidden rotational symmetries. In 2002 Lifshitz $^{14}$ pointed out that quasicrystals are not exclusively defined in terms of possessing forbidden symmetries. He introduced the Fibonacci square grid, which exhibits four-fold symmetry, but is quasicrystalline ${ }^{14}$. The square grid is constructed by superimposing two orthogonal Fibonacci linear grids (Fig. 1a). The Fibonacci linear grid is produced using short $(S)$ and long $(L)$ sections and certain substitution rules: $S \rightarrow L$ and $L \rightarrow L S$. These conditions create a sequence: $S, L, L S, L S L, L S L L S \ldots$. When $L=\tau S$, where $\tau$ is the golden mean $\left(\frac{1+\sqrt{5}}{2}=1.618 \ldots\right)$, this sequence models a onedimensional quasicrystal. The Fibonacci square grid is constituted by three tiles $(S \times S, S \times L$ and $L \times L)$ highlighted in Fig. 1a by different colours. Like other complex aperiodic tilings, the Fibonacci square grid follows specific rules for tile placement and frequency, and exhibits $\tau$-inflation symmetry ${ }^{14}$, i.e., the enlargement or shrinking of dimensional quantities by $\tau$. The theoretical construct was later extended to a cubic Fibonacci tiling in three dimensions ${ }^{15}$. Lifshitz ${ }^{14}$ noted at the time that to the best of his knowledge, no alloys or real quasicrystals existed with the structure of the square or cubic Fibonacci tilings.

The physical properties of a hypothetical quasicrystal with the square Fibonacci grid structure have been explored theoretically, including its electronic, phononic and transport behaviours ${ }^{15-17}$. Dallapiccola et al. ${ }^{18}$ examined the plasmonic properties of a lithographically fabricated Fibonacci square grid of a sub- micrometre scale. Similarly, Vardeny et al. ${ }^{19}$ studied the photonic properties of a system with a stacking of slabs of two different periodic materials in a Fibonacci sequence, as an example of onedimensional quasicrystalline structure. In this work we present experimental observation of the Fibonacci square grid in a molecular overlayer system.

\section{Results}

Substrate structure. We used the surface of an intermetallic quasicrystal, icosahedral (i)-Al-Pd-Mn, as a template for $\mathrm{C}_{60}$ adsorption. The icosahedral quasicrystal possesses two-, threeand five-fold rotational symmetry axes. Here we have used a surface perpendicular to the two-fold axis. The substrate surface was prepared as explained in the Methods section. Low-energy electron diffraction (LEED) and scanning tunnelling microscopy (STM) were utilised to characterise the surface.

The analysis of $k$-vectors of the LEED pattern (Fig. 1b) reveals that the surface corresponds to a bulk termination (Supplementary Note 1). This means that the surface has two-fold symmetry, as expected from the bulk. Consistent with the LEED results, fast Fourier transforms (FFTs) of STM images from the clean surface are also two-fold (Fig. 2a, see further discussion later). STM images can also be explained as bulk atomic planes. The observation of two-fold symmetry of the surface is in agreement with previous STM results ${ }^{20,21}$ (Supplementary Note 2).

STM of $\mathrm{C}_{60}$ overlayer. When $\mathrm{C}_{60}$ is deposited on the clean surface at $600 \mathrm{~K}$, a quasicrystalline network is formed, as shown in Fig. 1c. A majority of the $\mathrm{C}_{60}$ molecules $(70 \%$ of the total observed) lie at the vertices of a Fibonacci square grid of $S=1.26$
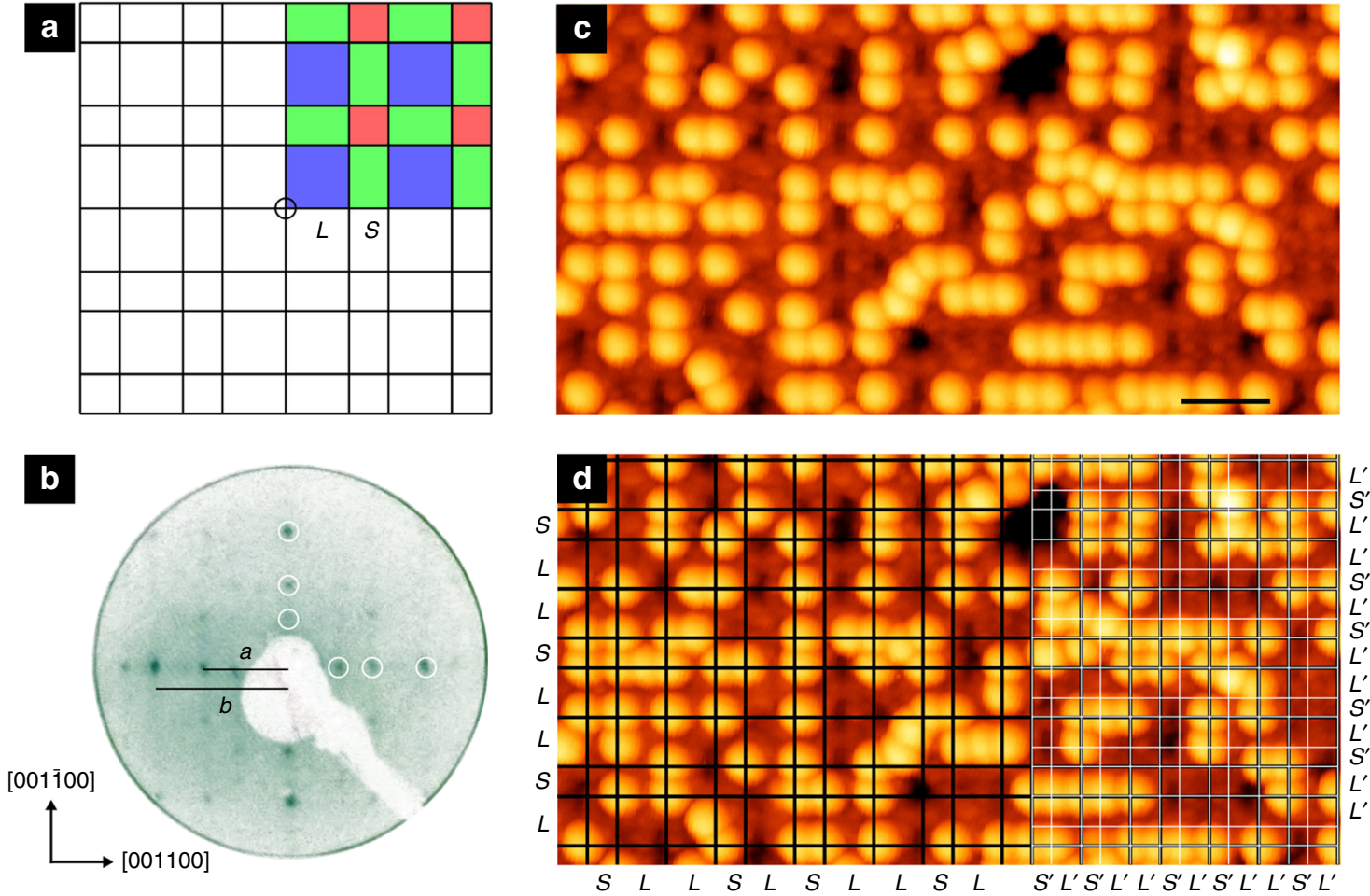

Fig. 1 Fibonacci square grid, LEED and STM. a The Fibonacci square grid, with highlighted constituent tiles (blue: $L \times L$, red: $S \times S$, and green: $S \times L$ ). The centre of rotation of four-fold symmetry is marked by a circle. $\mathbf{b}$ Low-energy electron diffraction pattern ( $60 \mathrm{eV}$, inverted for clarity) from the clean two-fold surface of the icosahedral (i) Al-Pd-Mn quasicrystal. Diffraction spots are $\tau$-scaled in the primary two-fold axes (highlighted with white circles), with two reciprocal space lengths indicated: $a=(14.1 \pm 0.3) \mathrm{nm}^{-1}$ and $b=(22.5 \pm 0.3) \mathrm{nm}^{-1}$. c Quasicrystalline $\mathrm{C}_{60}$ on the two-fold $i$-Al-Pd-Mn quasicrystal as imaged by scanning tunnelling microscopy. Scale bar represents $4 \mathrm{~nm}$. d Image c overlaid with a Fibonacci square grid of $S=1.26 \mathrm{~nm}, L=2.04 \mathrm{~nm}$ (black lines) to highlight the ordering of $C_{60}$. A $\tau$-deflated Fibonacci square grid of $L^{\prime}=L / \tau=S$ and $S^{\prime}=S / \tau$ is shown by white lines on the right-hand side. Note that the original grid overlaps with the $\tau$-deflated grid 

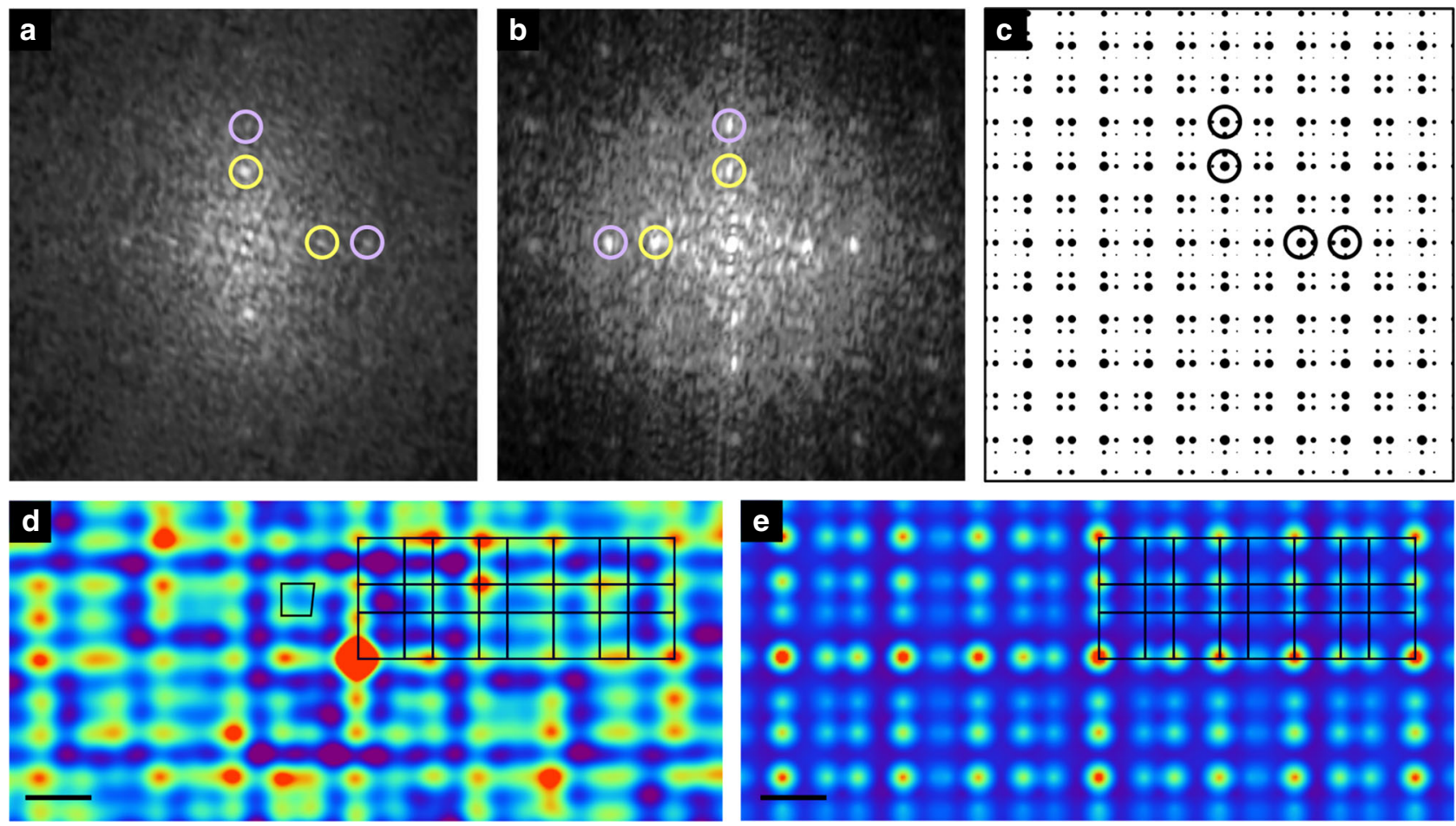

Fig. 2 Fast Fourier transform and autocorrelation function. a Fast Fourier transform (FFT) of a scanning tunnelling microscopy (STM) image of the clean surface, demonstrating two-fold symmetry. b FFT of the $\mathrm{C}_{60}$ molecules of Fig. 1c. c FFT generated from a Fibonacci square grid, showing four-fold symmetry. Spots of the first two orders of diffraction are highlighted by circles of different colours. FFTs in $\mathbf{a}$, $\mathbf{b}$ are displayed with the same $k$-vector scale, while the scale of $\mathbf{c}$ is arbitrary. $\mathbf{d}$ Autocorrelation function of the STM image of the $\mathrm{C}_{60}$ overlayer of Fig. 1c. A distorted square is marked. e Autocorrelation function taken from point objects at the vertices of a Fibonacci square grid of $S=1.26 \mathrm{~nm}, L=2.04 \mathrm{~nm}$. Scale bars in $\mathbf{d}$, e represent $1 \mathrm{~nm}$

$\mathrm{nm}, L=2.04 \mathrm{~nm}$. This grid is superimposed in Fig. 1d (black lines). The remaining minority of $\mathrm{C}_{60}$ can be placed at the vertices of a $\tau$-deflated grid. The $\tau$-deflated grid is shown by white lines in Fig. $1 \mathrm{~d}$, where $L^{\prime}=L / \tau=S$ and $S^{\prime}=S / \tau$. The origin of low occupancy of $\mathrm{C}_{60}$ at the $\tau$-deflated vertices will be discussed later.

We present FFT patterns taken from the clean and $\mathrm{C}_{60}$ dosed surfaces in Fig. 2a, b. For the calculation of the $\mathrm{C}_{60}$ pattern, the substrate was filtered out so that only the molecular film made a contribution to the FFT. Spots with equal $k$-vectors are highlighted by circles, indicating first- and second-order spots. The FFT spots for the substrate (Fig. 2a) and $\mathrm{C}_{60}$ (Fig. 2b) appear at the same $k$-vectors but their intensity distribution is different. The real space values corresponding to the $k$-vectors of FFTs are related to the length scales of $L$ and $S$.

The Fibonacci square grid structure of the $\mathrm{C}_{60}$ overlayer is confirmed by comparing the FFT of the STM image with the reciprocal space transform of the Fibonacci square grid (Fig. 2c). A grid of $S(=1.26 \mathrm{~nm})$ and $L(=2.04 \mathrm{~nm})$ lengths was chosen to allow for a direct comparison to the experimental FFT results. The pattern fits extremely well with the FFT of the $\mathrm{C}_{60}$ overlayer (Fig. 2c).

We also compare autocorrelation functions that are calculated from the STM image and from a model Fibonacci square grid. Figure $2 \mathrm{~d}$ is the autocorrelation function of the $\mathrm{C}_{60}$ molecules in the STM image shown in Fig. 1c. Similar to the FFT of the $\mathrm{C}_{60}$ molecules (Fig. 2b), the contribution from the substrate in STM was removed so that the autocorrelation function arises solely from the $\mathrm{C}_{60}$ molecules. Figure $2 \mathrm{e}$ shows the autocorrelation function of a perfect grid, i.e., a point object is placed at every vertex of a Fibonacci square grid of $S=1.26 \mathrm{~nm}$ and $L=2.04 \mathrm{~nm}$. The size of grid used to calculate the autocorrelation was $50 \mathrm{~nm} \times$ $50 \mathrm{~nm}$, but we have shown only a section for comparison. As expected, spots in the autocorrelation function of the model grid form a perfect Fibonacci square grid. In agreement with the model, the autocorrelation function of the STM image can also be mapped by a Fibonacci square grid. The tile lengths of the grid are also $S$ and $L$. This means that $\mathrm{C}_{60}$ molecules at the $\tau$-deflated grid do not contribute to the autocorrelation pattern. This is expected, as only a fraction of the $\tau$-deflated vertices are occupied by $\mathrm{C}_{60}$. In a perfect Fibonacci square grid, the number of vertices in the $\tau$-deflated grid is $\tau^{2}$ times the number of vertices in the original grid. The relative density of vertices of the $\tau$-deflated grid that do not overlap with the original grid is thus $\tau^{2}-1=\tau$, i.e., there are $\sim 162 \%$ more than the vertices of the original grid. However, only $\sim 8 \%$ of these vertices are occupied by $\mathrm{C}_{60}$ as observed by STM, and therefore these molecules do not produce additional features in the autocorrelation pattern.

\section{Discussion}

The specific adsorption sites of the molecules will now be considered. For this, we present the atomic structure of the two-fold surface of the $i$-Al-Pd-Mn quasicrystal in Fig. 3a, b. The structure corresponds to high-density planes of the bulk atomic model proposed by Boudard et al. ${ }^{22}$. The STM images of the substrate are consistent with these atomic planes (see Supplementary Note 2). Following direct measurement from the STM data (Fig. 1c) and comparison with theoretical autocorrelation results (Fig. 2e), the size of the $\mathrm{C}_{60}$ Fibonacci grid is known. This grid $(S=1.26 \mathrm{~nm}, L=2.04 \mathrm{~nm}$ ) fits with the separations of the $\mathrm{Mn}$ atoms in the surface plane (Fig. 3a, b), suggesting that individual $\mathrm{C}_{60}$ molecules adsorb directly on top of these Mn atoms. Aluminium adsorption sites are ruled out, as although $\mathrm{Al}$ atoms are separated by similar $S$ and $L$ lengths, the $\mathrm{Al}$ density at the surface would provide too many adsorption sites-producing a disordered film. This is also true for Pd sites. 

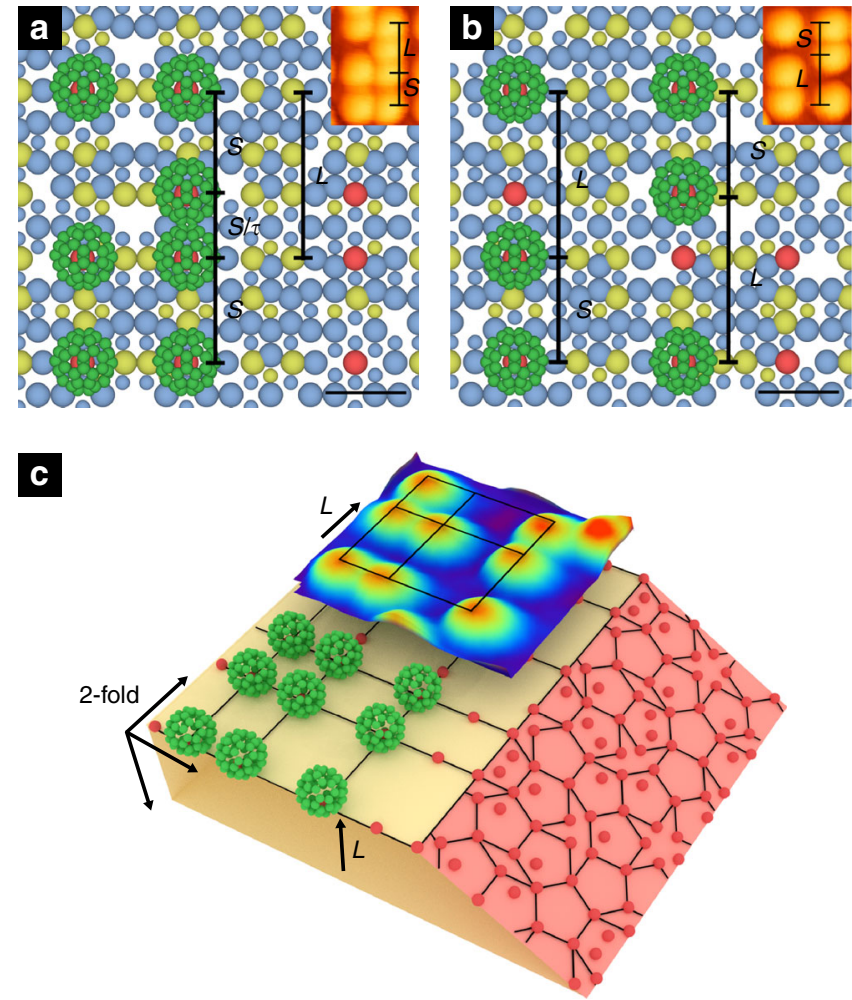

Fig. 3 Adsorption sites. a, b Comparison between $\mathrm{C}_{60}$ motifs observed by scanning tunnelling microscopy (STM) and the model structure of icosahedral (i)-Al-Pd-Mn. Model structures showing $\mathrm{C}_{60}$ adsorbed atop $M n$ atoms, forming $S \times S$ and $S \times L$ tiles. Two images are taken from two different sections of the surface plane in order to illustrate common STM features. The insets in $\mathbf{a}, \mathbf{b}$ are sections of the STM image from Fig. 1c. The inset in a shows an additional $\mathrm{C}_{60}$ adsorbed at a $\tau$-deflated position, which appears as a squashed (i.e., non-circular) molecule. $\mathrm{Al}=$ blue, $\mathrm{Pd}=$ yellow, $\mathrm{Mn}=$ red and $\mathrm{C}_{60}=$ green. Atoms in different layers are presented in different sizes, with the largest in the top layer. Scale bars in $\mathbf{a}, \mathbf{b}$ represent $1 \mathrm{~nm}$. c A block of the model structure of $\mathrm{i}-\mathrm{Al}-\mathrm{Pd}-\mathrm{Mn}$ displaying five-fold (pink) and two-fold (cream) planes, with $\mathrm{C}_{60}$ adsorbed atop $\mathrm{Mn}$ atoms. The orientation of $\mathrm{C}_{60}$ is arbitrary. Only $\mathrm{Mn}$ atoms are shown for clarity. There are three equivalent two-fold axes in icosahedral quasicrystal, which are orthogonal to each other: two of them are in the surface plane and the third is perpendicular to the surface plane. These directions are indicated by arrows. An STM image is superimposed for comparison

The Mn-based adsorption model is further strengthened by the Fibonacci square grid structure of the $\mathrm{C}_{60}$ overlayer and the substrate model structure. We present a block of the atomic structure of the $i$-Al-Pd-Mn quasicrystal, simultaneously displaying the five- and two-fold planes (Fig. 3c). For clarity we have shown only $\mathrm{Mn}$ atoms in the model. Mn atoms are located at the centre of pseudo-Mackay clusters, the building blocks of the $i$ Al-Pd-Mn quasicrystal ${ }^{23}$. The five-fold surface intersects the centre of the clusters and the Mn atoms can be mapped with a Penrose P1 tiling. The edge length of the tiling is $\sim 0.77 \mathrm{~nm}$, which is confirmed by $\mathrm{STM}^{24}$. The two-fold surface terminates at specific planes of the $\mathrm{P} 1$ tiling, such that $\mathrm{Mn}$ atoms form a Fibonacci square grid. A few remaining $\mathrm{Mn}$ atoms are located at $\tau$-deflated positions. Two of these planes are marked in Fig. 3c. However, Al and Pd atoms at the surface plane do not form a Fibonacci square grid. They show two-fold symmetry.

A comparison between the detailed structure of the $\mathrm{C}_{60}$ overlayer observed by STM, and Mn atomic positions in the model structure, also indicates that $\mathrm{Mn}$ atoms are the bonding sites. Figure 3a shows $S \times S$ and $S \times L$ tiles from the model whose vertices are decorated with $\mathrm{C}_{60}$. An additional $\mathrm{C}_{60}$ molecule occupies at an $S / \tau(0.77 \mathrm{~nm})$ deflated position. The inset on Fig. 3a is an STM image of the corresponding motif. In the model, the $S / \tau \mathrm{Mn}$ position is too close to its nearest Mn neighbour to allow neighbouring $\mathrm{C}_{60}$ molecules to adsorb without significant molecule-molecule interaction. The steric interaction between neighbouring $\mathrm{C}_{60}$ at these positions thus results in a slight spatial displacement away from the Mn adsorption sites. This displacement translates into the autocorrelation function, where some spots are displaced from the perfect Fibonacci grid producing deformed squares, see a marked distorted square in Fig. 2d. However, these $\tau$-deflated positions can also be occupied without $\mathrm{C}_{60}-\mathrm{C}_{60}$ displacement, as Fig. $3 \mathrm{~b}$ shows. Here, $\mathrm{C}_{60}$ molecules occupy Mn sites that create inverted Fibonacci sequences (again, inset is an STM motif). These positions may also contribute to the deformation of the experimental autocorrelation spots, as they simultaneously represent two inverted square grid tiles $(S \times S$ and $S \times L)$. In addition, the density of $\mathrm{Mn}$ atoms at $\tau$-deflated positions is very low in the model structure, compared to their density at the original grid. This is consistent with the low density of $\mathrm{C}_{60}$ at the $\tau$-deflated grid.

$\mathrm{C}_{60}$ is an excellent electron acceptor ${ }^{25}$ and $\mathrm{Mn}$ is electron rich $^{12}$. Therefore, a strong electronic molecule-substrate interaction at Mn sites is expected. Such behaviour was previously observed for $\mathrm{Bi}$ and $\mathrm{Si}$ on the five-fold surface of $i$-Al-Pd-Mn, where the adsorbates were observed to bond to $\mathrm{Mn}$ atoms ${ }^{9,26}$. Similarly, $\mathrm{C}_{60}$ was found to bond to $\mathrm{Fe}$ on the five-fold $i$ $\mathrm{Al}-\mathrm{Cu}-\mathrm{Fe}$ surface ${ }^{13}$. The $i-\mathrm{Al}-\mathrm{Cu}-\mathrm{Fe}$ quasicrystal and $i-$ Al-Pd-Mn have a very similar structure ${ }^{27}$.

In conclusion, we have shown that $\mathrm{C}_{60}$ molecules form a Fibonacci square grid on the two-fold surface of $i$-Al-Pd-Mn. The observation of such a structure extends the quasicrystal family beyond the forbidden symmetry systems previously observed. More generally, the sparse density of the minority constituents of complex metallic alloys presents a unique adsorption landscape for the construction of molecular arrangements on a chosen scale; for example, this methodology could permit the construction of a molecular magnet array of chosen magnetic behaviour. Additionally, understanding the propagation of waves inside five-, ten-, and twelve-fold quasicrystalline lattices as waveguides is an attractive problem due to the nearly isotropic Brillouin zones. However, it is a rather intractable one. A suitably constructed simplified Fibonacci square grid structure could therefore provide a useful stepping-stone to the understanding of complex phenomena such as the formation of photonic quasicrystals ${ }^{19}$. We have also shown that $\mathrm{C}_{60}$ can be utilised as a chemical probe that can tag unique adsorption sites of a complex structure, and thus provide an insight into the surface structure. This technique can of course be extended to other quasicrystalline and complex metallic alloy surface structures, providing that the experimental conditions are adequate; primarily the surface must have a unique or sparse enough adsorption network to provide meaningful topographic data.

\section{Methods}

Surface and thin film preparation. A two-fold Al-Pd-Mn quasicrystal was polished with successively finer grades of diamond paste from $6 \mu \mathrm{m}$ down to 0.25 $\mu \mathrm{m}$, before solvent washing with methanol in an ultrasonic bath. Upon insertion into ultra-high vacuum the sample was cleaned by several sputter-anneal cycles ( 30 min sputtering, $2 \mathrm{~h}$ anneal at $900 \mathrm{~K}$ ) to form a flat surface. Surface ordering and cleanliness was assessed with LEED. STM was used to investigate local atomic and molecular arrangements. $\mathrm{C}_{60}$ was deposited using thermal evaporation, while the substrate was held at $600 \mathrm{~K}$. The surface was exposed to the source at a rate of 0.1 monolayer per minute. A range of molecular coverages were investigated. 
Data availability. The main data supporting the findings of this study are included in this article and its Supplementary information files. Additional STM data are available from the corresponding author upon request.

Received: 27 March 2018 Accepted: 26 July 2018

Published online: 24 August 2018

\section{References}

1. Shechtman, D., Blech, I., Gratias, D. \& Cahn, J. W. Metallic phase with longrange orientational order and no translational symmetry. Phys. Rev. Lett. 53, 1951-1953 (1984).

2. Zeng, $X$. et al. Supramolecular dendritic liquid quasicrystals. Nature 428, 157-160 (2004).

3. Takano, A. et al. A mesoscopic Archimedean tiling having a new complexity in an ABC star polymer. J. Polym. Sci. Part B Polym. Phys. 43, 2427-2432 (2005).

4. Hayashida, K., Dotera, T., Takano, A. \& Matsushita, Y. Polymeric quasicrystal: mesoscopic quasicrystalline tiling in ABC star polymers. Phys. Rev. Lett. 98 195502 (2007).

5. Mikhael, J., Roth, J., Helden, L. \& Bechinger, C. Archimedean-like tiling on decagonal quasicrystalline surfaces. Nature 454, 501-504 (2008).

6. Fischer, S. et al. Colloidal quasicrystals with 12 -fold and 18-fold diffraction symmetry. Proc. Natl. Acad. Sci. USA 108, 1810-1814 (2011).

7. Förster, S., Meinel, K., Hammer, R., Trautmann, M. \& Widdra, W. Quasicrystalline structure formation in a classical crystalline thin-film system. Nature 502, 215 (2013).

8. Franke, K. J. et al. Quasicrystalline epitaxial single element monolayers on icosahedral Al-Pd-Mn and decagonal Al-Ni-Co quasicrystal surfaces. Phys. Rev. Lett. 89, 156104 (2002).

9. Smerdon, J. A. et al. Nucleation and growth of a quasicrystalline monolayer: $\mathrm{Bi}$ adsorption on the fivefold surface of $i-\mathrm{Al}_{70} \mathrm{Pd}_{21} \mathrm{Mn}_{9}$. Phys. Rev. B 78, 075407 (2008).

10. Smerdon, J. A. et al. Formation of a quasicrystalline $\mathrm{Pb}$ monolayer on the 10fold surface of the decagonal Al-Ni-Co quasicrystal. Surf. Sci. 602, 2496-2501 (2008).

11. Sharma, H. R. et al. Templated three-dimensional growth of quasicrystalline lead. Nat. Commun. 4, 2715 (2013).

12. Fournée, V. et al. Self-organized molecular films with long-range quasiperiodic order. ACS Nano 8, 3646-3653 (2014).

13. Smerdon, J. A. et al. Templated quasicrystalline molecular ordering. Nano. Lett. 14, 1184-1189 (2014)

14. Lifshitz, R. The square Fibonacci tiling. J. Alloys Compd. 342, 186-190 (2002).

15. Even-Dar Mandel, S. \& Lifshitz, R. Electronic energy spectra of square and cubic Fibonacci quasicrystals. Philos. Mag. 88, 2261-2273 (2008).

16. Ilan, R., Liberty, E., Mandel, S. E.-D. \& Lifshitz, R. Electrons and phonons on the square Fibonacci tiling. Ferroelectrics 305, 15-19 (2004).

17. Even-Dar Mandel, S. \& Lifshitz, R. Electronic energy spectra and wave functions on the square Fibonacci tiling. Philos. Mag. 86, 759-764 (2006).

18. Dallapiccola, R., Gopinath, A., Stellacci, F. \& Dal Negro, L. Quasi-periodic distribution of plasmon modes in two-dimensional Fibonacci arrays of metal nanoparticles. Opt. Express 16, 5544-5555 (2008).

19. Vardeny, Z. V., Nahata, A. \& Agrawal, A. Optics of photonic quasicrystals. Nat. Photonics 7, 177-187 (2013).

20. Reid, D., Smerdon, J. A., Ledieu, J. \& McGrath, R. The clean and copper-dosed two-fold surface of the icosahedral Al-Pd-Mn quasicrystal. Surf. Sci. 600, 4132-4136 (2006).

21. Gröning, O., Widmer, R., Ruffieux, P. \& Gröning, P. Scanning tunnelling microscopy with atomic resolution on the twofold surface of the icosahedral AlPdMn quasicrystal. Philos. Mag. 86, 773-779 (2006).
22. Boudard, M., Klein, H., de Boissieu, M., Audier, M. \& Vincent, H. Structure of quasicrystalline approximant phase in the AlPdMn system. Philos. Mag. A 74, 939-956 (1996).

23. Gratias, D., Puyraimond, F., Quiquandon, M. \& Katz, A. Atomic clusters in icosahedral F-type quasicrystals. Phys. Rev. B 63, 024202 (2000).

24. Papadopolos, Z. et al. Bulk termination of the quasicrystalline fivefold surface of Al-Pd-Mn. Phys. Rev. B 66, 184207 (2002).

25. Haddon, R. C., Palmer, R. E., Kroto, H. W. \& Sermon, P. A. The fullerenes: powerful carbon-based electron acceptors [and discussion]. Philos. Trans. Phys. Sci. Eng. 343, 53-62 (1993).

26. Ledieu, J., Unsworth, P., Lograsso, T. A., Ross, A. R. \& McGrath, R. Ordering of Si atoms on the fivefold Al-Pd-Mn quasicrystal surface. Phys. Rev. B 73, 012204 (2006).

27. Quiquandon, M. \& Gratias, D. Unique six-dimensional structural model for Al-Pd-Mn and Al-Cu-Fe icosahedral phases. Phys. Rev. B 74, 214205 (2006).

\section{Acknowledgements}

This work was supported by the Engineering and Physical Sciences Research Council (grant number EP/D071828/1). Partial support from the European Integrated Centre for the Development of New Metallic Alloys and Compounds is also acknowledged. The authors would like to thank M. Shimoda from the National Institute for Materials Science, Tsukuba, Japan for supplying the programme to analyse the surface atomic structure of the Al-Pd-Mn quasicrystal.

\section{Author contributions}

H.R.S. proposed the project. S.C. performed the STM and LEED experiments, analysed the data and drafted the manuscript. All authors contributed to discussion and interpretation of the data and to the manuscript. H.R.S. and R.M. supervised the whole project.

\section{Additional information}

Supplementary Information accompanies this paper at https://doi.org/10.1038/s41467018-05950-7.

Competing interests: The authors declare no competing interests.

Reprints and permission information is available online at http://npg.nature.com/ reprintsandpermissions/

Publisher's note: Springer Nature remains neutral with regard to jurisdictional claims in published maps and institutional affiliations.

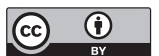

Open Access This article is licensed under a Creative Commons Attribution 4.0 International License, which permits use, sharing, adaptation, distribution and reproduction in any medium or format, as long as you give appropriate credit to the original author(s) and the source, provide a link to the Creative Commons license, and indicate if changes were made. The images or other third party material in this article are included in the article's Creative Commons license, unles indicated otherwise in a credit line to the material. If material is not included in the article's Creative Commons license and your intended use is not permitted by statutory regulation or exceeds the permitted use, you will need to obtain permission directly from the copyright holder. To view a copy of this license, visit http://creativecommons.org/ licenses/by/4.0/.

(c) The Author(s) 2018 